\title{
Prevalence of depression and its associated factors among adolescents in China during the COVID-19 outbreak
}

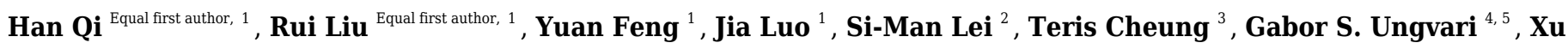 \\ Chen ${ }^{\text {Corresp., } 1 \text {, Yu-Tao Xiang }}{ }^{\text {Corresp. } 6,7}$ \\ ${ }^{1}$ The National Clinical Research Center for Mental Disorders \& Beijing Key Laboratory of Mental Disorders, Beijing Anding Hospital \& the Advanced \\ Innovation Center for Human Brain Protection, Capital Medical University, Beijing, China \\ 2 Faculty of Education, University of Macau, Macao SAR, China \\ 3 School of Nursing, Hong Kong Polytechnic University, Hong Kong SAR, China \\ 4 Division of Psychiatry, School of Medicine, University of Western Australia, Perth, Australia \\ 5 The University of Notre Dame Australia, Fremantle, Australia \\ 6 Unit of Psychiatry, Institute of Translational Medicine, Faculty of Health Sciences, University of Macau, Macao SAR, China \\ 7 Center for Cognition and Brain Sciences, University of Macau, Macao SAR, China \\ Corresponding Authors: Xu Chen, Yu-Tao Xiang \\ Email address: chenxuadyy@ccmu.edu.cn, ytxiang@um.edu.mo
}

Background: The outbreak of the 2019 coronavirus disease pandemic (COVID-19) had a detrimental impact on adolescents' daily life and studying, which could increase the risk of depression. This study examined the prevalence of depressive symptoms (depression hereafter) among Chinese adolescents and its associated factors.

Methods: An online survey was conducted during the COVID-19 outbreak in China. Adolescents aged 11-20 years who currently lived in China were invited to participate in the study. Data were collected with the "SurveyStar" platform using the Snowball Sampling method. Depression was assessed using the Center for Epidemiologic Studies Depression Scale (CES-D).

Results: A total of 9,554 adolescents participated in the study. The prevalence of depression was $36.6 \%$ (95\% Cl: 35.6\%-37.6\%); the prevalence of mild and moderate-severe depression was $9.2 \%(95 \% \mathrm{Cl}$ :

$8.9 \%-9.5 \%)$ and $27.4 \%$ (95\% Cl: $26.9 \%-27.9 \%)$, respectively. Female gender (OR=1.235, $\mathrm{P}<0.001)$, senior secondary school grade $(\mathrm{OR}=1.513, \mathrm{P}<0.001)$, sleep duration of $<6$ hours/day $(\mathrm{OR}=2.455, \mathrm{P}<0.001)$, and living in Hubei province (total number of infected cases $>10,000)(\mathrm{OR}=1.245, \mathrm{P}=0.038)$ were significantly associated with higher risk of depression. Concern about COVID-19 (OR=0.632, $P<0.001)$, participating in distance learning $(\mathrm{OR}=0.728, \mathrm{P}=0.001)$, sleep duration of $>8$ hours/day $(\mathrm{OR}=0.726$, $\mathrm{P}<0.001)$, exercise of $>30$ minutes/day, and study duration of $>4$ hours/day $(O R=0.835, P<0.001)$ were associated with lower risk of depression.

Conclusion: Depression was common among adolescents in China during the COVID-19 outbreak. Considering the negative impact of depression on daily life and health outcomes, timely screening and appropriate interventions are urgently needed for depressed adolescents during the COVID-19 pandemic. 
1 Prevalence of depression and its associated factors among adolescents in

4

\section{China during the COVID-19 outbreak}

Running title: depression of adolescents during COVID-19

Han $\mathrm{Qi}^{1 \#}$, Rui Liu ${ }^{1 \#}$, Yuan Feng ${ }^{1}$, Jia Luo ${ }^{1}, \mathrm{Si}_{-M a n}$ Lei $^{2}$, Teris Cheung ${ }^{3}$, Gabor S. Ungvari ${ }^{4,5}, \mathrm{Xu}$ Chen ${ }^{1}$, Yu-Tao Xiang6,7

1. The National Clinical Research Center for Mental Disorders \& Beijing Key Laboratory of Mental Disorders, Beijing Anding Hospital \& the Advanced Innovation Center for Human Brain Protection, Capital Medical University, Beijing, China;

2. Faculty of Education, University of Macau, Macao SAR, China;

3. School of Nursing, Hong Kong Polytechnic University, Hong Kong SAR, China;

4. Division of Psychiatry, School of Medicine, University of Western Australia, Perth, Australia;

5. The University of Notre Dame Australia, Fremantle, Australia;

6. Unit of Psychiatry, Institute of Translational Medicine, Faculty of Health Sciences, University of Macau, Macao SAR, China;

7. Center for Cognition and Brain Sciences, University of Macau, Macao SAR, China

\# These authors contributed equally to the work.

Corresponding Author:

Dr. Xu Chen ${ }^{1}$

The National Clinical Research Center for Mental Disorders \& Beijing Key Laboratory of Mental Disorders, Beijing Anding Hospital \& the Advanced Innovation Center for Human Brain Protection, Capital Medical University, Beijing, China.

Email address: chenxuadyy@ccmu.edu.cn.

Professor Yu-Tao Xiang 6,7

3/F, Building E12, Faculty of Health Sciences, University of Macau, Avenida da Universidade, Taipa, Macau SAR, China.

Email address: ytxiang@um.edu.mo. 
34

\section{Abstract}

Background: The outbreak of the 2019 coronavirus disease pandemic (COVID-19) had a detrimental impact on adolescents' daily life and studying, which could increase the risk of depression. This study examined the prevalence of depressive symptoms (depression hereafter) among Chinese adolescents and its associated factors.

Methods: An online survey was conducted during the COVID-19 outbreak in China. Adolescents aged 11-20 years who currently lived in China were invited to participate in this study. Data were collected with the "SurveyStar" platform using the Snowball Sampling method. Depression was assessed using the Center for Epidemiologic Studies Depression Scale (CES-D).

Results: A total of 9,554 adolescents participated in this study. The prevalence of depression was $36.6 \%$ (95\% CI: $35.6 \%-37.6 \%$ ); the prevalence of mild and moderate-severe depression was $9.2 \%$ (95\%CI: $8.9 \%-9.5 \%)$ and $27.4 \%$ (95\%CI: 26.9\%-27.9\%), respectively. Female gender $(\mathrm{OR}=1.235, \mathrm{P}<0.001)$, senior secondary school students $(\mathrm{OR}=1.513, \mathrm{P}<0.001)$, sleep duration of $<6$ hours/day $(\mathrm{OR}=2.455, \mathrm{P}<0.001)$, and living in Hubei province (total number of infected cases $>10,000)(\mathrm{OR}=1.245, \mathrm{P}=0.038)$ were significantly associated with higher risk of depression. Concern about COVID-19 (OR=0.632, $\mathrm{P}<0.001)$, participating in distance learning $(\mathrm{OR}=0.728$, $\mathrm{P}=0.001)$, sleep duration of $>8$ hours/day $(\mathrm{OR}=0.726, \mathrm{P}<0.001)$, exercise of $>30$ minutes/day, and study duration of $>4$ hours/day $(\mathrm{OR}=0.835, \mathrm{P}<0.001)$ were associated with lower risk of depression.

Conclusion: Depression was common among adolescents in China during the COVID-19 outbreak. Considering the negative impact of depression on daily life and health outcomes, timely screening and appropriate interventions are urgently needed for depressed adolescents during the disease pandemic.

Key words: Adolescents, China, COVID-19, depression, prevalence. 


\section{Introduction}

61 In December 2019, the coronavirus disease 2019 (COVID-19) outbreak started in Wuhan, Hubei

62 province, China, and then spread rapidly to more than 100 countries. On the $11^{\text {th }}$ of March 2020 ,

63 the World Health Organization (WHO) declared COVID-19 as a pandemic (World Health 64 Organization 2020).

65 With the rapid transmission of the COVID-19, mental health problems have been more

66 common in different segments of the population, such as confirmed / suspected cases, frontline medical professionals, and the elderly (Xiang et al. 2020; Yang et al. 2021; Yang et al. 2020). However, little is known about mental health of adolescents, although this is arguably a vulnerable subpopulation to experience mental health problems, particularly depression, due to academic pressure (Li \& Zhang 2014), negative life events (Li 2016), and low self-esteem (Lian et al. 2016). To reduce the rapid transmission, mass quarantine has been adopted and all face-to face school teaching in schools has been suspended in China during the COVID-19 pandemic. Distance learning and online teaching have been adopted for adolescents nationwide. Considering the uncertainty, fear, boredom, loneliness and anger associated with quarantine, challenges brought up by the sudden changes of traditional learning methods coupled with academic pressures, conflicts with parents, reduced social interaction with peers, and lack of outdoor activities, the risk of depressive symptoms (depression hereafter) was expected to increase among adolescents during the COVID-19 pandemic.

Several surveys on the prevalence of depression in adolescents have been conducted in China during the COVID-19 outbreak yielding conflicting findings: prevalence figures ranged from 6.41\% (Tang \& Pang 2020), 10.4\% (Wang et al. 2020c), 11.88\% (Chen et al. 2020), 21.16\% (Chang et al. 2020) to $43.7 \%$ (Zhou et al. 2020). Commonly reported correlates of depression during the pandemic included female gender, high school grade, decreased frequency of physical exercise, overuse of the internet and social media and familial vulnerability (Guessoum et al. 2020; Kilincel et al. 2021). The discrepancy of the findings between studies could be partly due to 
86

87

88

89

90

91

92

93

94

95

96

97

98

99

100

101

102

103

104

105

106

107

108

109

110

111

different sampling methods, rating instruments (e.g., the Patient Health Questionnaire (PHQ-9), the Depression Self-Rating Scale for Children (DSRS-C), and the Children's Depression Inventory (CDI)) and studies conducted at different stages of the COVID-19 outbreak. Many of these studies were completed after the peak of the COVID-19 outbreak (i.e., after February 2020), therefore their findings could not reflect the true spectrum of adolescents' mental health at the beginning of the outbreak(Chen et al. 2020; Zhou et al. 2020). Furthermore, nationwide data at the early stage of the outbreak were rarely reported (Tang \& Pang 2020; Wang et al. 2020c). To bridge the gap in knowledge of the impact of COVID-19 on adolescents, an online survey was conducted to investigate the prevalence of depression and exploring its associated factors in a large cohort of secondary school students in China in the early stage of COVID-19 pandemic.

\section{Materials \& Methods}

\section{Study design and participants}

This was an online survey conducted between February $20^{\text {th }}$ and $27^{\text {th }}, 2020$ within the context of the collaborative research network of the National Clinical Research Center for Mental Disorders, China (Qi et al. 2020). Data were collected with the WeChat-based "SurveyStar" program (https://www.wjx.cn/). Snowball sampling was used. The WeChat program has been widely used on student management in most secondary schools in China. To be eligible, participants should be: (1) secondary school students aged between 11 and 20 years; and (2) currently living in mainland China during the COVID-19 epidemic. Participants with severe mental disorders based on health records at schools or those who refused to participate were excluded in this study. All participants were required to provide written informed consent. For those under 18 years old, participants' legal guardians were also required to provide written consent. This study was approved by the Medical Ethical Committee in Beijing Anding Hospital of the Capital Medical University, China ((2020)KEYAN(No.10)). 
112

113

114

115

116

117

118

119

120

121

122

123

124

125

126

127

128

129

130

131

132

133

134

135

136

137

\section{Assessment instruments and data collection}

A data sheet designed for this study was used to collect socio-demographic and clinical characteristics, such as gender, age, place of residence, school grade, parents' occupations (frontline workers or not), attitude towards COVID-19, duration of physical exercise, attendance at distance learning, and presence of depression. Study sites were classified according to the total number of COVID-19 patients at provincial level based on the report of the National health commission of China (http://www.nhc.gov.cn) released on the Feb 27, 2020.

The presence of depression was assessed by the Chinese version of the Center for Epidemiological Studies Depression Scale (CES-D) (Radloff 1977). The CES-D is a 20-item selfreported questionnaire with satisfactory psychometric properties in Chinese adolescents (Chen et al. 2009). Each item is scored from 0 (not at all) to 3 (a lot). Participants with a CES-D total score of $>15$ was considered as 'depressed' (Zhang et al. 2010); those with a CES-D total score of 1619 were considered 'mildly depressed', and a CES-D total score of $>19$ indicated 'moderate to severe depression' (Gao 2009).

\section{Statistical analyses}

SPSS 21.0 was used to analyze data. Chi-square tests were used to compare demographic characteristics between depression and 'non depression' groups. Multivariate logistic regression analyses with the 'enter' method was performed to examine independent correlates of depression, with depression as the dependent variable, and those having significant group differences in univariate analyses as independent variables. The level of significance was 0.05 (two-tailed).

\section{Results}

With the exception of Tibet, all provinces of mainland China participated in this study. A total of 9,744 adolescents were invited to participate, of whom, 9,554 met the study criteria and were included in the analyses. The overall prevalence of depression was 36.6\% (95\%CI: 35.6\%-37.6\%), 
138

139

140

141

142

143

144

145

146

147

148

149

150

151

152

153

154

155

156

157

158

159

160

161

162

163

with the prevalence of mild and moderate-severe of $9.2 \%$ (95\% CI: $8.9 \%-9.5 \%)$ and $27.4 \%$ (95\%CI: $26.9 \%-27.9 \%)$, respectively.

Table 1 shows the socio-demographic and clinical characteristics comparing the depression and 'non-depression' groups. Univariate analyses revealed that age, gender, place of residence, school grade, infected acquaintances, attitudes toward COVID-19, sleep duration, duration of daily physical exercise, study time, and distant learning were significantly associated with depression $(\mathrm{P}<0.05)$. Figure 1 and 2 presents the CES-D total scores according to school grades and duration of physical exercise.

Table 2 shows the results of multivariate logistic regression analyses. Females $(\mathrm{OR}=1.235$, 95\% CI: 1.130-1.348, $\mathrm{P}<0.001)$, and senior secondary school students ( $\mathrm{OR}=1.513,95 \%$ CI: 1.379 1.661, $\mathrm{P}<0.001)$, sleep duration $<6$ hours/day ( $\mathrm{OR}=2.455,95 \% \mathrm{CI}: 1.998-3.016, \mathrm{P}<0.001)$, and living in Hubei province (total number of infected cases $>10,000)(\mathrm{OR}=1.245,95 \%$ CI: 1.013 $1.530, \mathrm{P}=0.038$ ) were significantly associated with higher risk of depression. In contrast, students who concerned about the COVID-19 (OR=0.632, 95\% CI: 0.568-0.703, $\mathrm{P}<0.001$ ), sleep duration $>8$ hours/day $(\mathrm{OR}=0.726,95 \% \mathrm{CI}$ : $0.662-0.796, \mathrm{P}<0.001)$, participating in distance learning $(\mathrm{OR}=0.728,95 \% \mathrm{CI}: 0.608-0.873, \mathrm{P}=0.001)$, duration of physical exercise $>30 \mathrm{~min} / \mathrm{day}$, and that of study $\geq 4$ hours/day ( $\mathrm{OR}=0.835,95 \% \mathrm{CI}: 0.740-0.943, \mathrm{P}<0.001)$ were significantly associated with lower risk of depression.

\section{Discussion}

To the best of our knowledge, this was the first survey examining the prevalence of depression and its associated factors among adolescents during the COVID-19 outbreak. The main finding of the survey is that $36.6 \%$ (95\% CI: $35.6 \%-37.6 \%$ ) of Chinese adolescents suffered from depression as defined by a score of $>15$ on the self-reported CES-D; of whom $27.4 \%$ presented with moderate to severe depression (95\% CI: $26.9 \%-27.9 \%)$. In China, there were 148.4 million adolescents in 2018 (National Bureau of Statistics of China et al. 2018), suggesting that approximately 54.3 
164 million adolescents could be suffering from broadly defined depression extrapolating the results 165 of this study.

166 A recent meta-analysis found that the pooled prevalence of depression in secondary school 167 students in mainland China was 24.3\% (95\% CI: 21.3\%-27.6\%) (Tang et al. 2019). Compared to 168 Tang et al.'s findings (Tang et al. 2019), the current study figures were higher, indicating that the 169 COVID-19 outbreak was a possibly significant risk factor contributing to the development of depression among adolescents. The anxiety-provoking experience brought about by the COVID19 outbreak, such as mass quarantine, limited public transportation, fear of infection, frustration, boredom, anger, lack of interpersonal contact with peers, limited personal space at home, financial loss in the family, uncertainty about the future were highly likely associated with the growing risk of depression (Brooks et al. 2020; Wang et al. 2020b; Wang et al. 2016).

Consisted with previous studies (Leung et al. 2020; Zhang et al. 2020; Zhou et al. 2020), female students were more likely to suffer from depression. The gender difference in the prevalence of depression could be partly attributed to endocrine and neurodevelopmental during the pubertal transition (Hyde et al. 2008) and more frequent introvert and vulnerable personality traits in girls (Salk et al. 2017). During the COVID-19 outbreak, all primary and secondary schools in China were temporally closed to reduce the rapid COVID-19 transmission. Students were instructed to stay at home, reduce visits to others and limit outdoor activities. Nevertheless, increased physical exercise, sleep and study duration were associated with lower risk of depression. Other studies confirmed that physical exercise (Carter et al. 2016), and sleeping up to 8-9 hours/day (Chiu et al. 2018; Guo et al. 2017) reduce the likelihood of depression in adolescents. The present study also confirmed previous findings (Guo et al. 2017; Raniti et al. 2017) that sleeping for less than 8 hours was associated with increased risk of depression.

Distance learning was associated with decreased risk of depression, which may be attributed to regular study schedules under teachers' supervision (Guo et al. 2017). Senior secondary school students had higher risk of depression than junior students. Compared to other countries, secondary 
190

191

192

193

194

195

196

197

198

199

200

201

202

203

204

205

206

207

208

209

210

211

212

213

214

215

school students in China may have higher study pressure from schools and parents, which may exacerbate the risk of depression (Tang et al. 2020). A recent study suggested that Chinese educational authorities should issue guidelines on effective online learning, provide online education on healthy lifestyle and online psychosocial support programmes (Wang et al. 2020b) to relieve the study pressure on adolescents.

Adolescents living in Hubei province had a higher risk of depression than other areas of China. Hubei province was probably the original epicenter of the COVID-19 outbreak in China. Therefore, a wide range of strict quarantine measures were immediately adopted to prevent rapid transmission of infection to other provinces. Prolonged quarantine has negative impact on mental health (Brooks et al. 2020), which could explain the higher risk of depression in the current study. Additionally, the large number of infected cases and deaths in Hubei province undoubtedly created an atmosphere of intense fear in the community elevating the risk of mental health problems, particularly depression among the residents.

Students who were more concerned about the COVID-19 had lower risk of depression, indicating that clear communication, regular and accurate updates about the COVID-19 reduced uncertainty and fear, and consequently the likelihood of depression (Xiang et al. 2020). Since the COVID-19 outbreak, daily press release by health authorities have disseminated update information about the epidemic in many regions of China (Wang et al. 2020a). The National Health Commission of China also released national guidelines of psychological crisis intervention related to the COVID-19 (Kang et al. 2020). Consequently, students' knowledge and better understanding about the COVID-19 epidemic were likely to substantially reduce their uncertainty and fear (Li \& Reavley 2021).

The strengths of this study included the large sample size covering almost all major areas of China. However, several limitations needed to be addressed. First, the design of our online survey meant that am unknown number of students without access to the internet could not participate in this study. However, the likelihood of not having internet access must have been very low, because 
216 of the near universal delivery of online teaching in schools in China. Second, relevant factors

217 associated with depression, such as peer social support, economic status, academic performance,

218 and relationships with families, could not be examined due to logistic reasons. Lastly, causality

219 between variables could not be examined due to the cross-sectional design of this study.

220

\section{Conclusions}

222 Depression was common among adolescents during the COVID-19 pandemic. Considering the 223 negative impact of depression on activities of daily life and health outcomes, timely screening and 224 appropriate interventions, such as online psychological counselling, are urgently needed to reduce

225 the likelihood of depression among adolescents during the current COVID-19 epidemic as well as 226 in similar possibly future infectious disease outbreaks.

227

228 Acknowledgements

229 N/A.

230

\section{Conflict of interest}

232 The authors declare no conflicts of interest. 
235

236

237

238

239

240

241

242

243

244

245

246

247

248

249

250

251

252

253

254

255

256

257

258

259

260

261

262

263

264

265

266

267

268

269

270

271

272

273

\section{References}

Brooks SK, Webster RK, Smith LE, Woodland L, Wessely S, Greenberg N, and Rubin GJ. 2020. The psychological impact of quarantine and how to reduce it: rapid review of the evidence. Lancet 395:912-920. 10.1016/s01406736(20)30460-8

Carter T, Morres ID, Meade O, and Callaghan P. 2016. The Effect of Exercise on Depressive Symptoms in Adolescents: A Systematic Review and Meta-Analysis. J Am Acad Child Adolesc Psychiatry 55:580-590. 10.1016/j.jaac.2016.04.016

Chang J, Yuan Y, and Wang D. 2020. Mental health status and its influencing factors among college students during the epidemic of COVID-19 (in Chinese). Journal of Southern Medical University 40:171-176. 10.12122/j.issn.1673-4254.2020.02.06

Chen F, Zheng D, Liu J, Gong Y, Guan Z, and Lou D. 2020. Depression and anxiety among adolescents during COVID-19: A cross-sectional study. Brain Behav Immun 88:36-38. 10.1016/j.bbi.2020.05.061

Chen ZY, Yang XD, and Li XY. 2009. Psychometric Features of CES-D in Chinese Adolescents (in Chinese). Chinese Journal of Clinical Psychology 17:443-445+448. CNKI:SUN:ZLCY.0.2009-04-019

Chiu HY, Lee HC, Chen PY, Lai YF, and Tu YK. 2018. Associations between sleep duration and suicidality in adolescents: A systematic review and dose-response meta-analysis. Sleep Med Rev 42:119-126. 10.1016/j.smrv.2018.07.003

Gao ZL. 2009. Research on Depression of Postgraduates in a Certain Famous University in Shanghai (in Chinese). Chinese Journal of Health Psychology 17:961-963. 10.13342/j.cnki.cjhp.2009.08.040

Guessoum SB, Lachal J, Radjack R, Carretier E, Minassian S, Benoit L, and Moro MR. 2020. Adolescent psychiatric disorders during the COVID-19 pandemic and lockdown. Psychiatry Res 291:113264. 10.1016/j.psychres.2020.113264

Guo L, Xu Y, Deng J, Huang J, Huang G, Gao X, Li P, Wu H, Pan S, Zhang WH, and Lu C. 2017. Association between sleep duration, suicidal ideation, and suicidal attempts among Chinese adolescents: The moderating role of depressive symptoms. $J$ Affect Disord 208:355-362. 10.1016/j.jad.2016.10.004

Hyde JS, Mezulis AH, and Abramson LY. 2008. The ABCs of depression: integrating affective, biological, and cognitive models to explain the emergence of the gender difference in depression. Psychol Rev 115:291-313. 10.1037/0033-295X.115.2.291

Kang L, Li Y, Hu S, Chen M, Yang C, Yang BX, Wang Y, Hu J, Lai J, and Ma X. 2020. The mental health of medical workers in Wuhan, China dealing with the 2019 novel coronavirus. The Lancet Psychiatry 7:e14.

Kilincel S, Kilincel O, Muratdagi G, Aydin A, and Usta MB. 2021. Factors affecting the anxiety levels of adolescents in home-quarantine during COVID-19 pandemic in Turkey. Asia Pac Psychiatry 13:e12406. 10.1111/appy. 12406

Leung DYP, Mak YW, Leung SF, Chiang VCL, and Loke AY. 2020. Measurement invariances of the PHQ-9 across gender and age groups in Chinese adolescents. Asia Pac Psychiatry 12:e12381. 10.1111/appy.12381

Li HL, and Zhang WX. 2014. Peer support: easing up the relationship between adolescents' learning pressure and depression (in Chinese). Chinese Journal of Special Education:87-91.

Li W, and Reavley N. 2021. Patients' and caregivers' knowledge and beliefs about mental illness in mainland China: A systematic review. Asia Pac Psychiatry 13:e12423. 10.1111/appy.12423 
274

275

276

277

278

279

280

281

282

283

284

285

286

287

288

289

290

291

292

293

294

295

296

297

298

299

300

301

302

303

304

305

306

307

308

309

310

311

312

Li YZ. 2016. Stress life events and suicide ideation among high school students: a mediated moderation model (in Chinese). Chinese Journal of Clinical Psychology 24:129-133+138. 10.16128/j.cnki.1005-3611.2016.01.030

Lian SL, Sun XJ, Tian Y, Yan JL, Niu GF, and Tong YT. 2016. The effect of adolescents' peer attachment on depression: the mediating role of friends social support and self-esteem (in Chinese). Journal of Psychological Science 39:1116-1122. 10.16719/j.cnki.1671-6981.20160515

National Bureau of Statistics of China, UNICEF China, and UNFPA China. 2018. Population Status of Adolescents in China in 2015: Facts and Figures (in Chinese). p 1-11. https://www.unicef.cn/en/reports/population-statuschildren-china-2015.

Qi H, Liu R, Chen X, Yuan XF, Li YQ, Huang HH, Zheng Y, and Wang G. 2020. Prevalence of anxiety and associated factors for Chinese adolescents during the COVID-19 outbreak. Psychiatry Clin Neurosci 74:555-557. 10.1111/pcn.13102

Radloff L. 1977. The CES-D scale: A self-report depression scale for research in the general population. Appl Psychol Meas:385-401. 10.1177/014662167700100306

Raniti MB, Allen NB, Schwartz O, Waloszek JM, Byrne ML, Woods MJ, Bei B, Nicholas CL, and Trinder J. 2017. Sleep Duration and Sleep Quality: Associations With Depressive Symptoms Across Adolescence. Behav Sleep Med 15:198-215. 10.1080/15402002.2015.1120198

Salk RH, Hyde JS, and Abramson LY. 2017. Gender differences in depression in representative national samples: Meta-analyses of diagnoses and symptoms. Psychol Bull 143:783-822. 10.1037/bul0000102

Tang S, and Pang HW. 2020. Anxiety and depression in children and adolescents during the COVID-19 epidemic (in Chinese). Mental Health Education in Primary and Secondary School:15-18. CNKI:SUN:ZXXK.0.2020-19005

Tang X, Tang S, Ren Z, and Wong DFK. 2019. Prevalence of depressive symptoms among adolescents in secondary school in mainland China: A systematic review and meta-analysis. J Affect Disord 245:498-507. 10.1016/j.jad.2018.11.043

Tang X, Tang S, Ren Z, and Wong DFK. 2020. Psychosocial risk factors associated with depressive symptoms among adolescents in secondary schools in mainland china: A systematic review and meta-analysis. $J$ Affect Disord 263:155-165. 10.1016/j.jad.2019.11.118

Wang C, Horby PW, Hayden FG, and Gao GF. 2020a. A novel coronavirus outbreak of global health concern. The Lancet 395:470-473. 10.1016/S0140-6736(20)30185-9

Wang GH, Zhang YT, Zhao J, Zhang J, and Jiang F. 2020b. Mitigate the effects of home confinement on children during the COVID-19 outbreak. The Lancet 395:945-947. 10.1016/S0140-6736(20)30547-X

Wang L, Feng Z, Yang G, Yang Y, Wang K, Dai Q, Zhao M, Hu C, Zhang R, Liu K, Guang Y, and Xia F. 2016. Depressive symptoms among children and adolescents in western china: An epidemiological survey of prevalence and correlates. Psychiatry Res 246:267-274. 10.1016/j.psychres.2016.09.050

Wang Y, Yang YY, li SW, Lei XM, and Yang YF. 2020c. Investigation on the status and influencing factors for depression symptom of children and adolescents with home quarantine during the prevalence of novel coronavirus peumonia (in Chinese). Chinese Journal of Child Health Care 28:277-280.

World Health Organization. 2020. WHO Director-General's opening remarks at the media briefing on COVID-19 11 March 2020. https://www.who.int/dg/speeches/detail/who-director-general-s-opening-remarks-at-the- 
313

314

315

316

317

318

319

320

321

322

323

324

325

326

327

328

329

330

331

media-briefing-on-covid-19---11-march-2020. (accessed Mar 11, 2020).

Xiang YT, Yang Y, Li W, Zhang L, Zhang Q, Cheung T, and Ng CH. 2020. Timely mental health care for the 2019 novel coronavirus outbreak is urgently needed. Lancet Psychiatry 7:228-229. 10.1016/s22150366(20)30046-8

Yang T, Chen J, Lam RW, Fang Y, and Xu Y. 2021. Mental Health Service Challenges during the Early Stage of the COVID-19 Pandemic: Experience and Best Practices from China. Can J Psychiatry 66:621-623. 10.1177/0706743720972252

Yang Y, Li W, Zhang Q, Zhang L, Cheung T, and Xiang YT. 2020. Mental health services for older adults in China during the COVID-19 outbreak. Lancet Psychiatry 7:e19. 10.1016/S2215-0366(20)30079-1

Zhang J, Wu ZY, Fang G, Li J, Han BX, and Chen ZY. 2010. Development of the Chinese age norms of CES-D in urban area (in Chinese). Chinese Mental Health Journal 24:139-143. CNKI:SUN:ZXWS.0.2010-02-019

Zhang Z, Zhai A, Yang M, Zhang J, Zhou H, Yang C, Duan S, and Zhou C. 2020. Prevalence of Depression and Anxiety Symptoms of High School Students in Shandong Province During the COVID-19 Epidemic. Front Psychiatry 11:570096. 10.3389/fpsyt.2020.570096

Zhou SJ, Zhang LG, Wang LL, Guo ZC, Wang JQ, Chen JC, Liu M, Chen X, and Chen JX. 2020. Prevalence and socio-demographic correlates of psychological health problems in Chinese adolescents during the outbreak of COVID-19. Eur Child Adolesc Psychiatry 29:749-758. 10.1007/s00787-020-01541-4

Peer] reviewing PDF | (2021:02:57919:1:1:REVIEW 7 Aug 2021) 
333 Figure Legends

334

335 Figure 1. The CES-D mean scores and standard errors by different grades of adolescents during 336 the COVID-19 outbreak.

337

338

Figure 2. The CES-D mean scores and standard errors by physical activities of adolescents during 339 the COVID-19 outbreak. 


\section{Figure 1}

The CES-D mean scores and standard errors by different grades of adolescents during the COVID-19 outbreak.

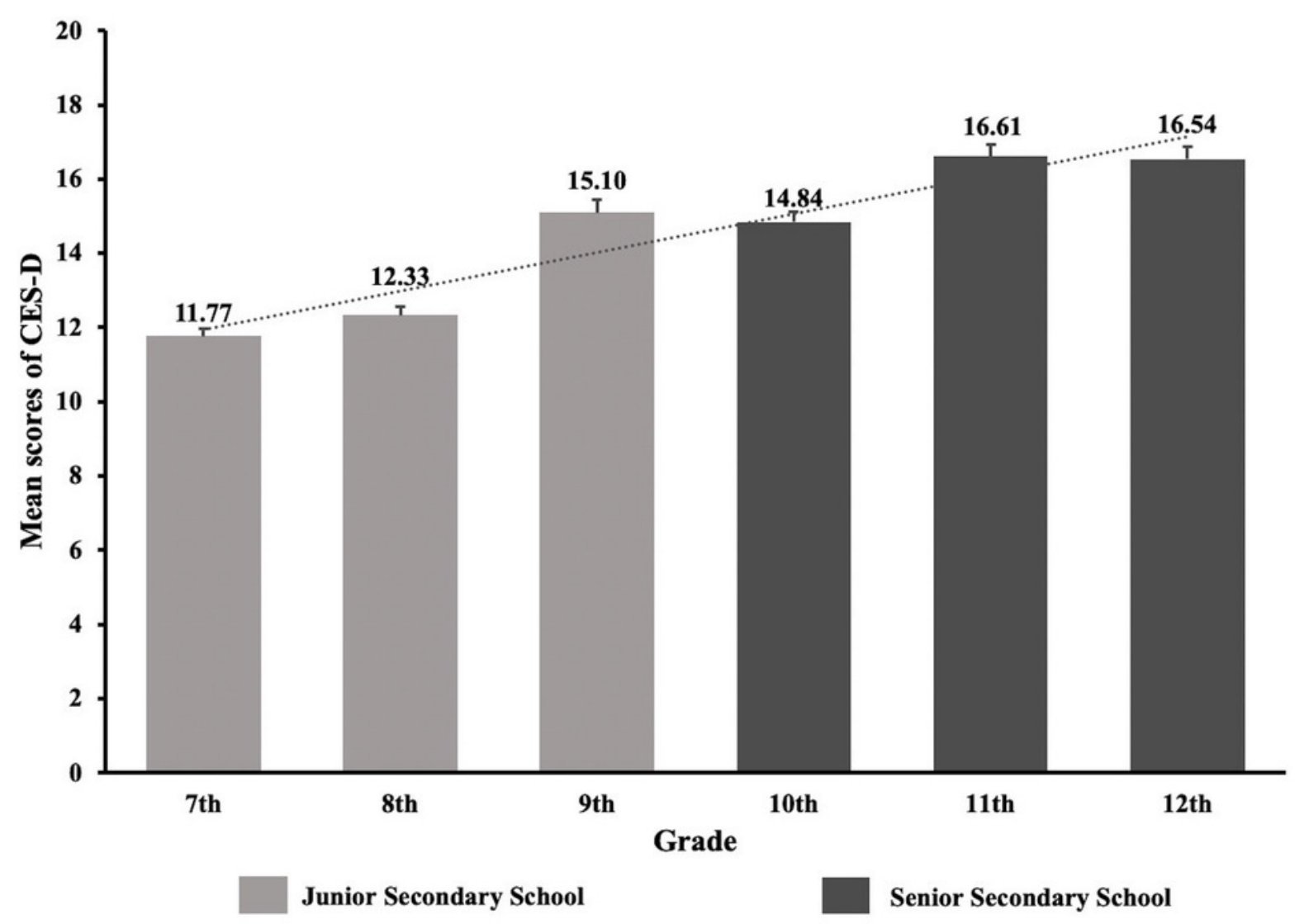




\section{Figure 2}

Figure 2. The CES-D mean scores and standard errors by physical activities of adolescents during the COVID-19 outbreak.

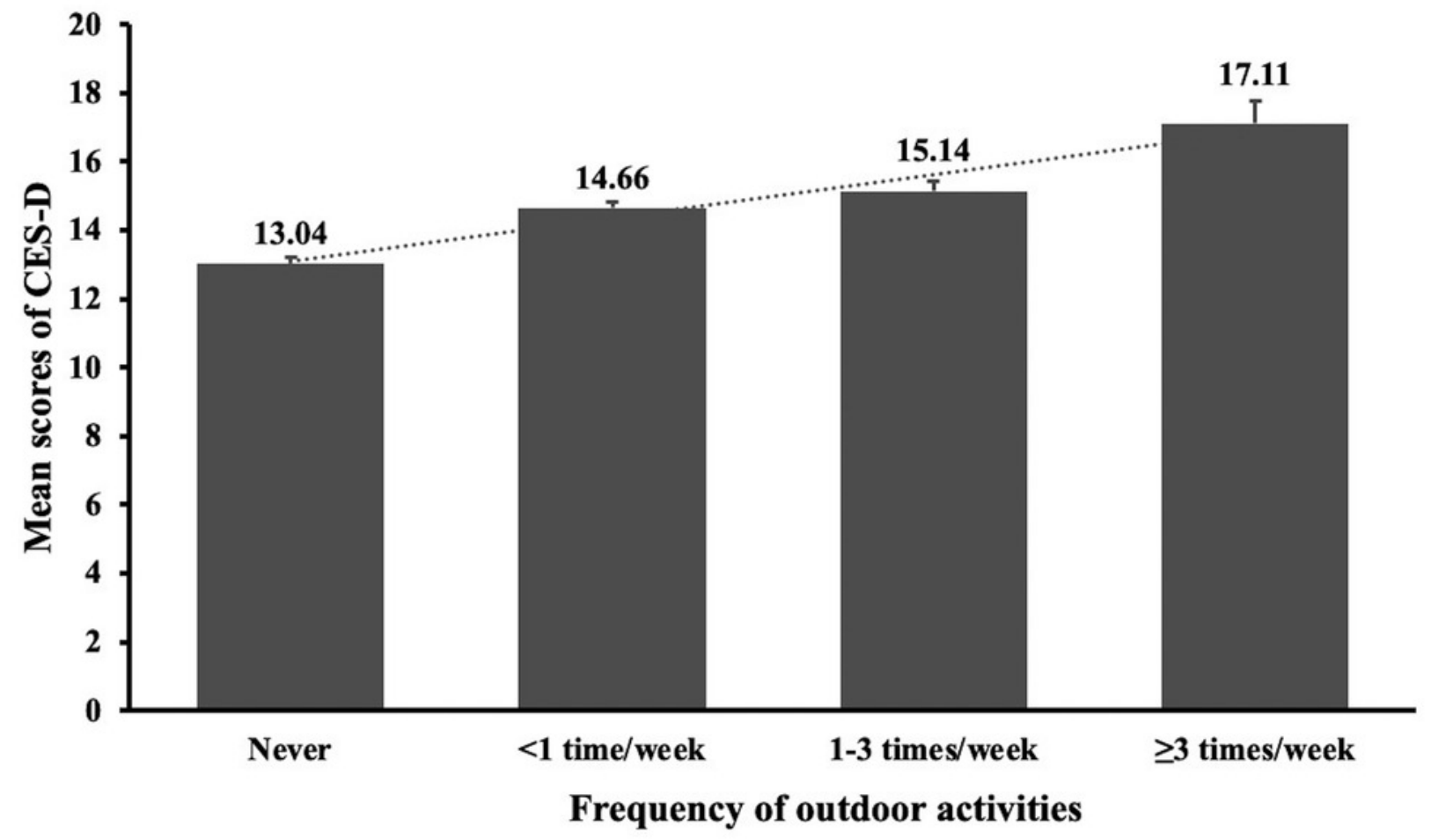




\section{Table $\mathbf{1}$ (on next page)}

Demographic characteristics of the study sample $(N=9,554)$ 
1 Table 1. Demographic characteristics of the study sample $(\mathrm{N}=9,554)$ 2

\begin{tabular}{|c|c|c|c|c|c|c|c|c|}
\hline \multirow[t]{2}{*}{ Variables } & \multicolumn{2}{|c|}{$\begin{array}{c}\text { Total } \\
(\mathrm{N}=9,554)\end{array}$} & \multicolumn{2}{|c|}{$\begin{array}{l}\text { Depression } \\
(\mathrm{N}=\mathbf{3}, 498)\end{array}$} & \multicolumn{2}{|c|}{$\begin{array}{c}\text { No depression } \\
(\mathrm{N}=6,056)\end{array}$} & \multirow[t]{2}{*}{$x^{2}$} & \multirow[t]{2}{*}{ P-value } \\
\hline & $\mathbf{n}$ & $\%$ & $\mathbf{n}$ & $\%$ & $\mathbf{n}$ & $\%$ & & \\
\hline Age (years) & & & & & & & 176.33 & $<0.001$ \\
\hline $11-15$ & 5,607 & 58.7 & 1,745 & 49.9 & 3,862 & 63.8 & & \\
\hline $16-20$ & 3.947 & 41.3 & 1,753 & 50.1 & 2,194 & 36.2 & & \\
\hline Male gender & 4,577 & 47.9 & 1,514 & 43.3 & 3,063 & 50.6 & 47.30 & $<0.001$ \\
\hline $\begin{array}{l}\text { Having infected } \\
\text { acquaintances }\end{array}$ & 336 & 3.5 & 143 & 4.1 & 193 & 3.2 & 5.31 & 0.021 \\
\hline $\begin{array}{l}\text { Parents as frontline } \\
\text { workers }\end{array}$ & 823 & 8.6 & 312 & 8.9 & 511 & 8.4 & 0.65 & 0.419 \\
\hline $\begin{array}{l}\text { Be concerned about } \\
\text { COVID-19 }\end{array}$ & 7,639 & 80.0 & 2580 & 73.8 & 5,059 & 83.5 & 132.35 & $<0.001$ \\
\hline Study duration $\geq 4 \mathrm{~h} /$ day & 8,012 & 83.9 & 2,794 & 79.9 & 5,218 & 86.2 & 64.78 & $<0.001$ \\
\hline Distance learning & 8,953 & 93.7 & 3,181 & 90.9 & 5,772 & 95.3 & 71.92 & $<0.001$ \\
\hline Grade & & & & & & & 172.10 & $<0.001$ \\
\hline Junior secondary school & 5,459 & 57.1 & 1,693 & 48.4 & 3,766 & 62.2 & & \\
\hline Senior secondary school & 4,095 & 42.9 & 1,805 & 51.6 & 2,290 & 37.8 & & \\
\hline $\begin{array}{l}\text { Living area (No. of } \\
\text { infected patients) }\end{array}$ & & & & & & & 8.72 & 0.033 \\
\hline $10-99$ & 2,224 & 23.3 & 825 & 23.6 & 1,399 & 23.1 & - & - \\
\hline $100-999$ & 5,177 & 54.2 & 1,932 & 55.2 & 3245 & 53.6 & - & - \\
\hline $1,000-9,999$ & 1,631 & 17.1 & 545 & 15.6 & 1,086 & 17.9 & - & - \\
\hline$>10,000$ & 522 & 5.5 & 196 & 5.6 & 326 & 5.4 & & \\
\hline Sleep duration/day & & & & & & & 221.30 & $<0.001$ \\
\hline$<6 h$ & 446 & 4.7 & 283 & 8.1 & 163 & 2.7 & - & - \\
\hline $6-8 \mathrm{~h}$ & 5,359 & 56.1 & 2,089 & 59.7 & 3,270 & 54.0 & - & - \\
\hline$>8 \mathrm{~h}$ & 3,749 & 39.2 & 1,126 & 32.2 & 2,623 & 43.3 & & \\
\hline $\begin{array}{l}\text { Physical exercise } \\
\text { duration/day (indoor and } \\
\text { outdoor) }\end{array}$ & & & & & & & 181.55 & $<0.001$ \\
\hline$<30 \mathrm{~min}$ & 4,392 & 46 & 1,924 & 55 & 2,468 & 40.8 & - & - \\
\hline $30-60 \mathrm{~min}$ & 4,250 & 44.5 & 1,289 & 36.8 & 2,961 & 48.9 & - & - \\
\hline$>60 \mathrm{~min}$ & 912 & 9.5 & 285 & 8.1 & 627 & 10.4 & & \\
\hline Residence & & & & & & & 2.05 & 0.152 \\
\hline Dorm & 74 & 0.8 & 33 & 0.9 & 41 & 0.7 & - & - \\
\hline Others & 9,480 & 99.2 & 3,465 & 99.1 & 6,015 & 99.3 & & \\
\hline
\end{tabular}


3 Note: Provinces were classified according to the number of confirmed cases of COVID-19

4 for where the students lived; Depression was defined as total score of the Center for

5 Epidemiological Studies Depression Scale for Children (CES-D) >15; COVID-19: Corona

6 Virus Disease 2019; Bolded values: $\mathrm{P}<0.05$. 
Table 2 (on next page)

Table 2. Independent correlates of depression by multivariate logistical regression analysis 
1 Table 2. Independent correlates of depression by multivariate logistical regression 2 analysis.

\begin{tabular}{|c|c|c|c|c|}
\hline \multirow{2}{*}{ Variables } & \multirow{2}{*}{ P-value } & \multirow{2}{*}{$O R$} & \multicolumn{2}{|c|}{$95 \%$ CIs for OR } \\
\hline & & & Lower & Upper \\
\hline Female & $<0.001$ & 1.235 & 1.130 & 1.348 \\
\hline Senior secondary school & $<0.001$ & 1.513 & 1.379 & 1.661 \\
\hline $\begin{array}{l}\text { Having infected } \\
\text { acquaintances }\end{array}$ & 0.063 & 1.241 & 0.988 & 1.559 \\
\hline $\begin{array}{l}\text { Concerned about COVID- } \\
2019\end{array}$ & $<0.001$ & 0.632 & 0.568 & 0.703 \\
\hline \multicolumn{5}{|l|}{ Sleep duration/day } \\
\hline $6-8 h$ & ref & - & - & - \\
\hline$<6 \mathrm{~h}$ & $<0.001$ & 2.455 & 1.998 & 3.016 \\
\hline$>8 \mathrm{~h}$ & $<0.001$ & 0.726 & 0.662 & 0.796 \\
\hline \multicolumn{5}{|l|}{$\begin{array}{l}\text { Physical exercise } \\
\text { duration/day (indoor and } \\
\text { outdoor) }\end{array}$} \\
\hline$<30 \mathrm{~min}$ & ref & - & - & - \\
\hline $30-60 \mathrm{~min}$ & $<0.001$ & 0.636 & 0.580 & 0.698 \\
\hline$>60 \mathrm{~min}$ & $<0.001$ & 0.686 & 0.586 & 0.803 \\
\hline \multicolumn{5}{|l|}{ Study duration/day } \\
\hline$<4 \mathrm{~h}$ & ref & - & - & - \\
\hline$\geq 4 \mathrm{~h}$ & 0.004 & 0.835 & 0.740 & 0.943 \\
\hline Distance learning & 0.001 & 0.728 & 0.608 & 0.873 \\
\hline \multicolumn{5}{|l|}{$\begin{array}{l}\text { Living area (No. of } \\
\text { infected patients) }\end{array}$} \\
\hline $10-99$ & ref & - & - & - \\
\hline $100-999$ & 0.236 & 1.068 & 0.958 & 1.191 \\
\hline $1,000-9,999$ & 0.002 & 0.797 & 0.691 & 0.918 \\
\hline$>10,000$ & 0.038 & 1.245 & 1.013 & 1.530 \\
\hline
\end{tabular}

4 Note: Due to collinearity between age and grade, age group was not entered in the 5 multiple logistic regression analysis. Bolded values: $\mathrm{P}<0.05$; $\mathrm{CI}$ : confidential interval; OR: 6 odds ratio. 